\title{
Carcinosarcoma of the Hypopharynx-A Rare Entity with Unique Surgical Procedure
}

\author{
Sangeet Kumar Agarwal', Satinder Singh, Shalabh Sharma, Asish Kumar Lahiri \\ Department of ENT and Head, Neck Surgery, Sir Ganga Ram Hospital, New Delhi, India \\ Email: *drsangeetagarwal@gmail.com
}

Received August 12, 2013; revised September 10, 2013; accepted October 2, 2013

Copyright (C) 2013 Sangeet Kumar Agarwal et al. This is an open access article distributed under the Creative Commons Attribution License, which permits unrestricted use, distribution, and reproduction in any medium, provided the original work is properly cited.

\begin{abstract}
Background: Carcinosarcoma of hypopharynx is a rare neoplasm with both malignant epithelial and mesenchymal components. They are highly aggressive in nature with controversial histogenesis. To our knowledge, there is no case reported of carcinosarcoma of hypopharynx managed with wide LASER excision and modified radical neck dissection followed by chemoradiation. Method and Result: 42-year-old male presented complaints of foreign body sensation in throat, and difficulty in swallowing and hoarseness for 4 months. On examination, a large smooth globular mass filling the supraglottis obscuring the view of endolarynx was noted and right side level-II cervical lymphadenopathy was found. CECT neck revealed a large hypopharyngeal mass abutting the supraglottis. Microlaryngoscopic biopsy was reported as high grade carcinosarcoma and PET scan was done after 1 week which revealed no any distant metastasis. The patient was taken for transoral pulsed carbon dioxide LASER excision with modified radical neck dissection, and postoperative chemoradiation was done and after two years of closed follow-up MRI was done which revealed no disease and the patient is absolutely free of disease till now. Conclusion: It was a rare case which was managed with complete LASER excision with neck dissection and postoperative chemoradiation and the patient is absolutely free of disease till now after 2 years of follow-up.
\end{abstract}

Keywords: Carcinosarcoma; PET Scan; LASER

\section{Introduction}

Carcinosarcoma is a rare neoplasm with both malignant epithelial and malignant mesenchymal components. Carcinosarcoma of the head and neck is uncommon. Most reported cases have occurred in the major salivary glands; other sites include the larynx and pharynx and less frequently the oral and nasal cavities and the esophagus. True carcinosarcomas of the hypopharynx and larynx represent less than $1 \%$ of all malignant neoplasms in this area. Carcinosarcomas are aggressive regardless of their origin, but differentiating their origin is important because the prognosis also depends on the site of origin [1].

The histogenesis is controversial and the most accepted theory is the differentiation of primitive blastic mesenchymal cells that can mature and produce malignant neoplasm of multiple differentiations [2].

To our knowledge, there is no case reported on carcinosarcoma of hypopharynx managed with wide LASER excision and modified radical neck dissection followed

*Corresponding author. by chemoradiation.

\section{Case Report}

A 42 years old male presented with complaints of foreign body sensation in throat and hoarseness for last 4 months. He also had difficulty in deglutition, which was more to solids than to liquids for last 2 months. He was a chronic smoker, betel nut chewer and had a history of alcohol consumption regularly. He did not have difficulty in breathing at presentation. Rigid Hopkins Rod Laryngoscopy showed a large smooth globular mass filling the supra-glottis, endolarynx could not be seen and origin of the mass could not be made out. A single right side level II Cervical lymphnode was palpable which was approximately $1.5 \mathrm{~cm} \times 1.5 \mathrm{~cm}$ in size and firm in consistency was noted. Contrast Enhanced Computed Tomograhy scan of neck was done which revealed enhancing mass inseparable from the right aryepiglottic fold and the right posterolateral pharyngeal wall abutting the epiglottis with enlarged necrotic right upper deep cervical lymph 
nodes (Figure 1).

In view of the compromised airway, temporary tracheostomy followed by microlaryngoscopic excision biopsy was done using a Weerda distending suspension laryngoscope, the mass was found to be attached with a broad based pedicle to right pharyngoepiglottic fold going down upto superior border of lateral wall of pyriform sinus. The pedicle base was cauterized with a bipolar forceps prior to excision. Biopsy (S1607/11) was reported as high grade carcinosarcoma.

Gross pathological examination showed a single bulky nodular piece of tissue measuring $3 \times 2.5 \times 2 \mathrm{~cm}$ with smooth outer surface, showed focal areas of necrosis. Cut surface was grey white, lobulated and gelatinous.

Microscopically nodular tumor with largely ulcerated overlying epithelium was seen. The tumor shows biphasic pattern having both epithelial as well as stromal components. Epithelial component showed poorly differentiated squamous cell carcinoma in most places while in occasional foci, it showed squamous differentiation with keratin pearls. Spindle cell component showed prominent osteoid deposition in lace like pattern (deposited by tumor cells). Calcification was also prominent within the tumor along with focally scattered inflammatory cells. Few scattered giant cells are also seen. Mitosis was very high (25 - 30/10 HPF) with many atypical mitosis. Necrosis is confined in the periphery of the tumor.

Immunohistochemistry examination revealed that the epithelial component of the tumor cells were strongly positive of EMA and bcl2. Occasional cells stain Cytokeratin. Spindle cells are positive for vimentin. CD34 and S-100 stains are negative. SMA shows nuclear positivity in most of the cells (both epithelial and stromal)

(Figure 2).

PET scan was done after 1 week to rule out distant metastasis (Figure 3).

Patient was taken up for transoral laser excision of the base of the previously excised pedicle. The slough covered areas left behind after the excision biopsy were marked out using LASER with margin of $5 \mathrm{~mm}$. The edge of the area was held with forceps and pulled medially and dissected away from the muscular layer with the pulsed $\mathrm{CO}_{2}$ LASER. This included the pharyngo-epiglottic fold and the right edge of the epiglottis. Right modified radical neck dissection type I was done. Margins of the surgical resection were negative, out of total 44 neck nodes, 3 lymph nodes were invaded by tumor. Patient was referred for chemotherapy and radiotherapy.

Image guided intensity modulated radiation therapy (IGRT), total dose of 66 Gy was given in 33 fractions. He also received concurrent chemotherapy with inj.cisplatinum $70 \mathrm{mg}$ once a week for 6 weeks. He tolerated the CT and RT well. The latest Magnetic resonance imaging of neck done after 1.5 years reveals no evidence of

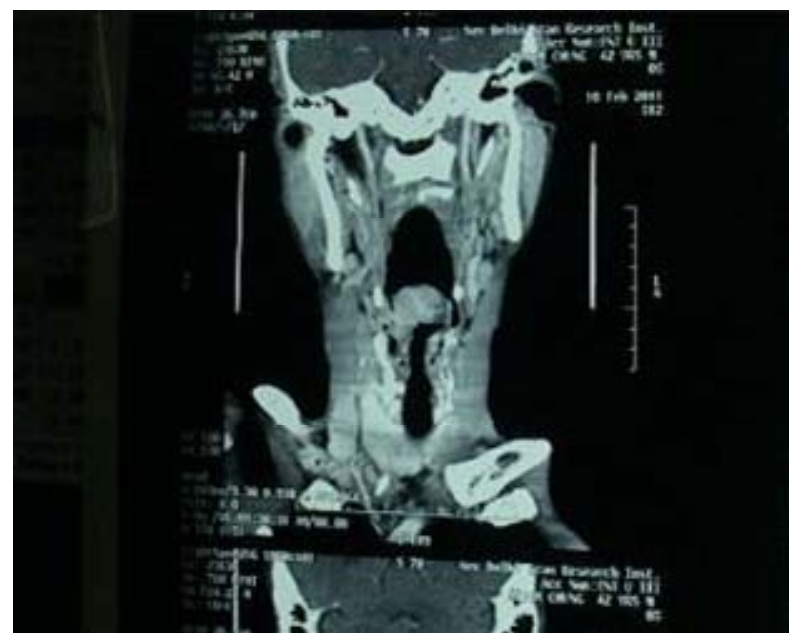

Figure 1. Coronal cut of CECT scan showing globular lesion arising from lateral pharyngeal wall obscuring the lumen.

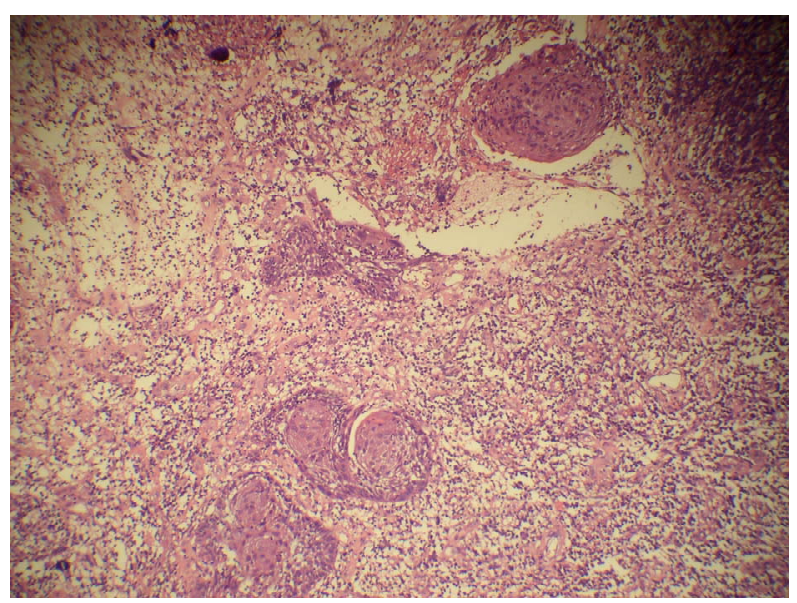

Figure 2. Histopathological slide initially stained with hematoxylin and eosin (H \& E) and finally stained with vimentin and S-100, showing epithelial and mesenchymal components.

disease remnant and patient is absolutely free of disease till now (Figure 4).

\section{Discussion}

Carcinosarcoma is an unusual and rare neoplasm of the upper aerodigestive tract. The diagnosis implies a biphasic neoplasm containing both mesenchymal and epithelial elements, each of which displays the histologic and biologic criteria of malignancy [2].

In 1865, Virchow named the rare malignant neoplasm of squmaous and sarcomatous cell types "carcinosarcoma”, since then it has also been called pseudosarcoma, spindle cell carcinoma and sarcomatoid carcinoma. This mixed type of tumour with a combination of carcinomatous and sarcomatous cell types has been found in cancers of the uterus, vagina, lungs, oral cavity, larynx, 


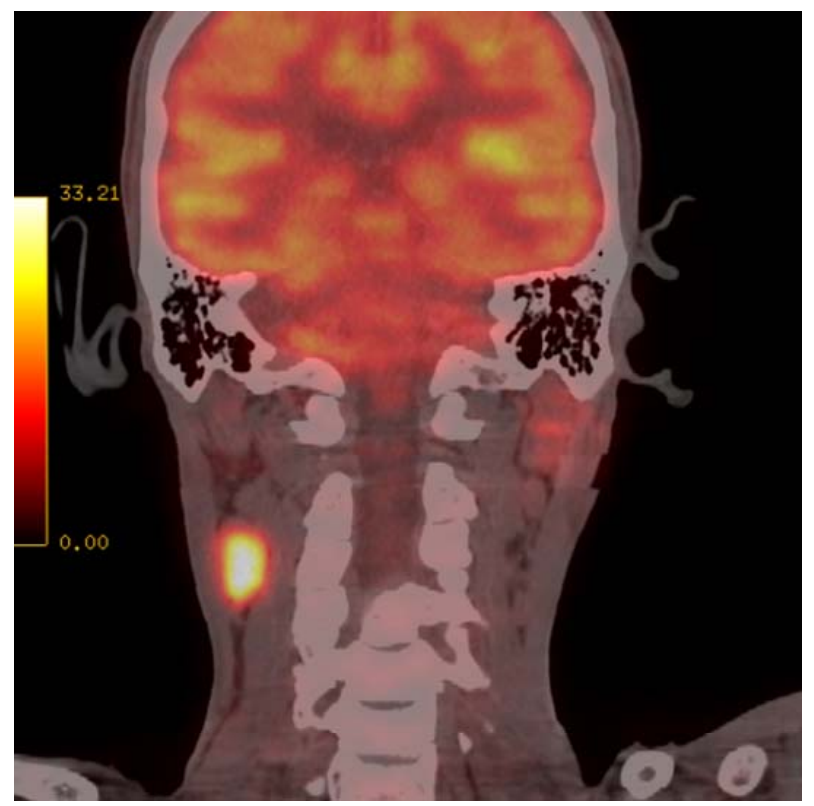

Figure 3. Coronal cut of PET-CT scan showing increased uptake of 18-FDG (fluro deoxy glucose) in left upper jugulodigastric lymphnode.

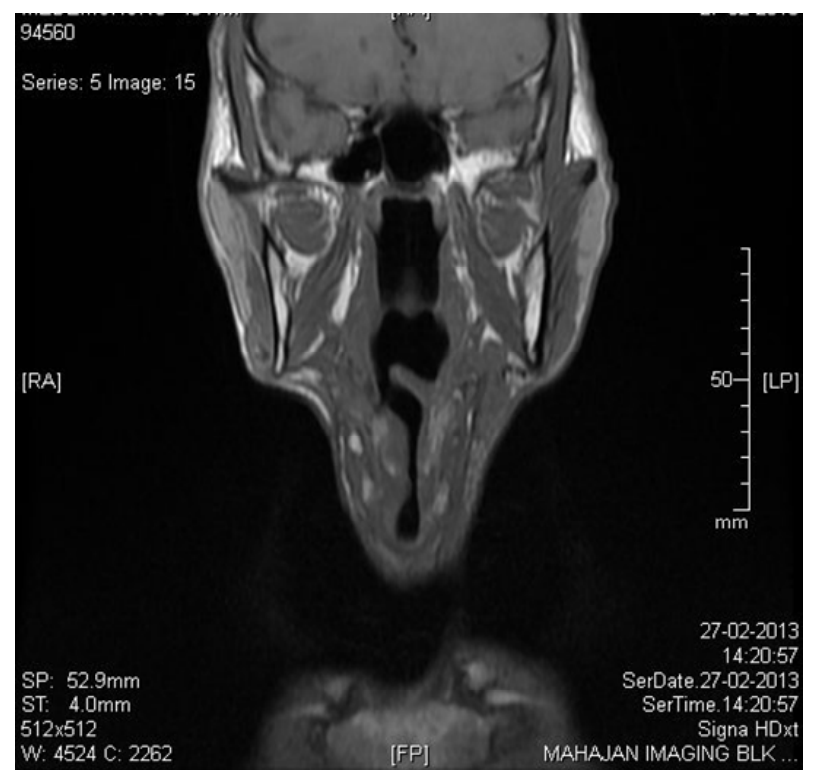

Figure 4. Coronal cut of contrast enhanced MRI showing post radiotherapy edema of the pharynx with partial absence of epiglottis without showing recurrence.

thyroid, urinary tract and esophagus [3,4].

This tumor is uncommon in the hypopharynx and is commoner elsewhere in the upper aerodigestive tract, and in decreasing order of frequency-larynx, oral cavity, hypopharynx \& pyriform fossa, sinonasal tract and oropharynx. It has an association with smoking (87\%), alcohol (48\%) and history of radiation therapy. The tumor may recur locally (18\%) or have distant metastasis (14\%) [5]. The lesion is a polypoidal, exophytic, or fungating mass. Surface ulceration is common with these tumors [6].

The exact histogenesis has been a source of debate. Four pathogenic explanations have been proposed. First, the tumor may be a carcinosarcoma, which is a collision tumor. Second, the tumor may be a squamous cell carcinoma with an atypical reactive stoma (pseudosarcoma). Third, a malignancy arises in an embryonic rest of epithelial and mesenchymal tissues. And fourth, the tumor de-differentiating results in spindle cell morphologic features (sarcomatoid carcinoma) [2].

In case of carcinosarcoma, there is primitive mesenchymal blastic cell differentiation that can mature and produce malignant neoplasms of multiple differentiations. True sarcoma is the major component with a high mitotic index, cell pleomorphism, and dysplasia to invasive carcinoma or adenocarcinoma, and no areas of transition are observed.

On immunohistochemistry, epithelial component is positive to cytokeratin, and mesenchymal component is positive to vimentin and other markers like S-100, actin, desmin etc. depending on tumour's origin.

In the present case, the epithelial component of the tumor cells is strongly positive to EMA and bcl2. Occasional cells stain cytokeratin. Spindle cells are positive to vimentin. CD34 and S-100 stains are negative. SMA shows nuclear positivity in most of the cells (both epithelial and stromal).

Diagnosis is usually established early on the basis of the clinical presentation which is generally characterized by the presence of an exophytic tumour that quickly causes obstruction as a result of its rapid growth, especially the growth of the mesenchymal component. A patient with suspected carcinosarcoma should undergo a deep biopsy of a non-ulcerated area because when ulceration is present, the affected area can become over infected which can lead to a false negative biopsy [1].

The differential diagnosis of spindle cell carcinoma includes rare sarcoma such as fibrosarcoma, malignant fibrous histiocytoma, and another biphasic tumor-synovial cell sarcoma. Also hypocellular reactive processes such as radiation changes or granulation tissue must be distinguished from spindle cell carcinoma. Immunostains for keratin are often useful in diagnosis. The presence of keratin positivity and nondiploid DNA content in the spindle cell population supports the neoplastic epithelial origin of these tumors (sarcomatoid carcinoma). The overall tumor behavior and surgical therapy appeared to be comparable with those of squamous cell carcinoma at a similar stage [2].

There is no consensus as to the most appropriate therapy for carcinosarcoma. The most common treatment is total laryngectomy complemented in some cases by adjuvant chemotherapy and/or radiotherapy because the 
prognosis is dependent on the type of malignant mesenchymal component. [7] In accordance with the previous literature, surgical therapy is the primary mode of treatment of these lesions. Conservational operations are performed when indicated. Various methods include conservation partial laryngectomies like vertical and horizontal partial laryngectomies and in some cases more radical surgeries like supracricoid partial laryngectomy with cricohyoidpexy and total laryngectomies with or without neck dissections are done primarily for hypopharyngeal, supraglottic, advanced glottic and transglottic tumors. Another mode of treatment is chemotherapy and radiotherapy alone or combined with surgery. Conservational surgeries and non-surgical therapy are functionally preservative methods but prognostically less favourable methods. As the metastasis has been reported for such type of carcinomas, it seems to be reasonable to use combined therapy, as neck dissections are associated with better cure rates, and elective neck dissection must be performed at the time of the initial treatment of a nonepithelial neoplasm, therefore the most common treatment is definitive surgery of that organ complemented with neck dissection in some cases by adjuvant chemotherapy and/or radiotherapy [1,7-9].

Prognosis of true carcinosarcoma of the hypopharynx is very poor because it is very aggressive and highly metastatic in nature, and most of the cases died due to distant metastasis to lungs. The exact prognosis of these tumours is not known.

The most important prognostic factors appear to be location \{hypopharynx versus larynx and supraglottic versus glottic\}, the size of the tumor and the presence of cervical metastasis.

In the present case, the tumour was excised from the hypopharynx with the help of pulsed $\mathrm{CO}_{2}$ LASER with modified radical neck dissection type 1 , and postoperative concurrent radiotherapy and chemotherapy were given.

\section{REFERENCES}

[1] K. Luna-Ortiz and A. M. Taylor, "Supracricoid Partial Laryngectomy as a Primary Treatment for Carcinosarcoma of the Larynx," Ear, Nose and Throat Journal, Vol. 85, No. 5, 2006, pp. 337-341.

[2] K. D. Olsen, J. E. Lewis and V. J. Suman, "Spindle Cell Carcinoma of the Larynx and Hypopharynx," Otolaryngology_Head and Neck Surgery, Vol. 116, No. 1, 1997, pp. 47-52. http://dx.doi.org/10.1016/S0194-5998(97)70351-6

[3] J. C. Bull and O. F. Grimes, "Pulmonary Carcinosarcoma," Chest, Vol. 65, No. 1, 1974, pp. 9-12. http://dx.doi.org/10.1378/chest.65.1.9

[4] J. T. Au, G. Sugiyama, H. Wang, A. Nicastri, D. Lee, W. Ko and V. Tak, "Carcinosarcoma of the Esophagus-A Rare Mixed Type of Tumour," Journal of Strength and Conditioning Research, Vol. 7, 2010, p. 7.

[5] P. R. Lambert, P. H. Ward and G. Berci, "Pseudosarcoma of the Larynx,” Archives of Otolaryngology, Vol. 106, 1980, pp. 700-708.

http://dx.doi.org/10.1001/archotol.1980.00790350042012

[6] G. Randall, W. A. Alonso and J. H. Ogura, "Spindle Cell Carcinoma [Pseudosarcoma] of the Larynx," Archives of Otolaryngology, Vol. 101, No. 1, 1975, pp. 63-66. http://dx.doi.org/10.1001/archotol.1975.00780300067018

[7] F. Ianniello, E. Ferri, E. Armato, et al., "Carcinosarcoma of the Larynx: Immnohistochemical Study, Clinical Considerations, Therapeutic Strategies,” Acta Otorhinolaryngologica Italica, Vol. 21, No. 3, 2001, pp. 192-197.

[8] D. Vie Vers, A. de Vito, K. Luna-Ortiz, et al., "Supracricoid Partial Laryngectomy for Non Squamous Cell Carcinoma of the Larynx," Journal of Laryngology \& Otology, Vol. 115, No. 5, 2001, pp. 388-392.

[9] U. Srinivasan and G. V. Talyalkar, "True Carcinosarcoma of the Larynx: A Case Report,” Journal of Laryngology \& Otology, Vol. 93, No. 10, 1979, pp. 1031-1035. http://dx.doi.org/10.1017/S002221510008806X 